\title{
Meteorological Drought Warning Research in Fujian Province, China during 1971-2016
}

\author{
Shanmei Li1 ${ }^{1}$, Xianmei Wang1, Chengliang Gao ${ }^{*}$, Xiaobing Ye ${ }^{2}$ \\ ${ }^{1}$ Pingtan Comprehensive Pilot Zone Meteorological Bureau of Fujian Province, Pingtan, China \\ ${ }^{2}$ Yongchun County Meteorological Bureau of Fujian Province, Quanzhou, China \\ Email: ${ }^{*} 461096027 @ q q . c o m$
}

How to cite this paper: Li, S. M., Wang, X M., Gao, C. L., \& Ye, X. B. (2019). Meteorological Drought Warning Research in Fujian Province, China during 1971-2016. Journal of Geoscience and Environment Protection, 7, 220-228.

https://doi.org/10.4236/gep.2019.711016

Received: August 21, 2019

Accepted: November 25, 2019

Published: November 28, 2019

Copyright (c) 2019 by author(s) and Scientific Research Publishing Inc. This work is licensed under the Creative Commons Attribution International License (CC BY 4.0).

http://creativecommons.org/licenses/by/4.0/

\begin{abstract}
This paper optimizes the meteorological drought assessment method in $\mathrm{Fu}$ jian Province, China, making it more suitable for long-term serial drought statistics. Using the precipitation data of Pingtan National Basic Weather Station from 1971 to 2016, according to the optimized meteorological drought assessment method, statistics Pingtan meteorological drought process, and its characteristics analysis; through the multi-model integrated forecasting super-aggregate method, the 2011-2014 Fujian precipitation reality and the European Center, T639, WRF, Japan and other numerical forecast products were used for comparative analysis. The use of multiple regression method was used to forecast precipitation, by the localized precipitation correction scheme. The early warning method of single station meteorological drought process based on precipitation reality and precipitation forecast is obtained, and the platformization idea is proposed.
\end{abstract}

\section{Keywords}

Meteorological Drought, Precipitation Correction, Early Warning Method, Business Platform

\section{Introduction}

Drought usually refers to a climatic phenomenon in which the total amount of fresh water is small, which is insufficient to meet human survival and economic development, and is one of the most serious climate disasters affecting human society. The types of drought recognized by the World Meteorological Organization include: meteorological drought, climate drought, atmospheric drought, agricultural drought, hydrological drought and water management drought. The three general definitions in China include meteorological drought, agricultural 
drought and hydrological drought. On November 1, 2006, the national standard "Meteorological Drought Grade" was implemented. The meteorological drought has a unified grade standard, and its evaluation method has also been standardized. On this basis, Fujian Province further institutionalized the local standard “Methodological Drought Assessment Method" (DB35/T1283-2012) (hereinafter referred to as local standard), defined: meteorological drought refers to the income and expenditure due to evaporation and precipitation during a certain period of time Unbalanced, water expenditure is greater than the water shortage caused by water income; meteorological drought process refers to the long-lasting period without effective rainfall days and the accumulated rainfall does not reach the continuous rainy process of the release index; no effective rainfall day refers to the day when the daily rainfall is $\leq 2 \mathrm{~mm}$ (DB35/T1283-2012). These provide direction for the operational warning of meteorological drought.

At present, the meteorological department's focus on drought is mainly distributed in its disaster characteristics, cause analysis and evaluation indicators (Li et al., 2012; Duan et al., 2012; Chen \& Zhao 2012; Liu \& Xiao, 2012), while the early warning research on drought focuses on the monitoring and early warning of agricultural drought, such as Yan et al. (2012) and Li (2012). The drought monitoring and early warning methods based on MODIS data were studied, while Du et al. (2012) used TRMM data for drought monitoring and tested its reliability. However, for the grass-roots meteorological departments, these satellite remote sensing data or other agricultural data and the models used are difficult to adapt to local meteorological drought monitoring and early warning, and the information is not easy to obtain.

Fujian Pingtan Comprehensive Experimental Zone is located in the eastern part of Fujian Province. It is the fifth largest island in China and the largest island in Fujian Province. The island is poor in water resources and weak in water supply system. It is one of the most severe meteorological droughts in Fujian. Taking Pingtan as an example, this paper uses the most simple and easy-to-obtain daily precipitation data to carry out necessary business improvement on the evaluation method of meteorological drought in Fujian, effectively utilize and correct numerical forecast products, and conduct research on forecasting and early warning of meteorological drought process. The early warning method will be platformized to realize the forecasting and warning operation of the meteorological drought process.

\section{Data and Methods}

\subsection{Data and Processing}

Daily precipitation data of Pingtan National Basic Weather Station from 1971 to 2016 (20 to 20 o'clock), and Precipitation observation data of 1950 automatic stations (taken from the AI file used by the business) in Fujian Province from 2011 to 2014 are included. The numerical product data used are the most frequently used in the daily business: ECMWF, T639, FJWRF, JMA (Japan) and 
NCEP_GFS. The initial field is taken at 08:00 and 20:00 (Beijing time).

Set 1950 automatic stations to the station to be interpolated, read and write the automatic station AI file as MICAPS format for easy viewing and statistics, some unreasonable values in automatic station observation, preliminary quality control, quality control rules: $1 \mathrm{~h}$ precipitation $[0.500 \mathrm{~mm}]$. After the initial quality control, further quality control: one station for three consecutive hours of rain is more than $20 \mathrm{~mm}$. Data that is rejected by QC is not written to the file or recorded as a missing value. For the 24-hour forecast, the 24-hour rainfall forecast for the previous day's value product from 20:00, if the aging $\leq 72$ hours, the above five numerical products are available; if $>72$ hours, only the first two forecasts (ECMWF, T639), the remaining numerical products are not enough time to adopt.

\subsection{Improvement of Drought Evaluation Methods}

The calculation method of meteorological drought process used in this paper is quoted from the local standard "Evaluation Method of Meteorological Drought" (DB35/T1283-2012) in Fujian Province. However, in the actual operation process, it was found that the Local Standard still has some incompleteness in processing the data of consecutive sequences. According to the principle "not connected to drought and not clearing", we have improved it to meet the operational requirements of meteorological drought warning, as follows:

The drought in summer and autumn and winter is the drought process around October 10. Because the standard of summer drought (16 - 25 days) is smaller than the standard of autumn and winter drought (31 - 50 days), that is, the number of consecutive drought days before October 10 should be even greater. The number of dry days is not clear so even during the dry season transition. The same is true for the transition from spring drought to summer drought. The drought in the transition from autumn to winter to spring will increase the standard, and the continuous rainless days in autumn and winter will be reduced to the number of consecutive drought days in the next season.

Except for summer, autumn and winter droughts, the time span of other three seasons or more droughts can reach the special drought level after entering the next season considering the continuous drought process spanning three seasons and above, so it is directly under the standard of not reducing the seasons. The definition of drought in the three seasons is the special drought level, and the release of rainfall is subject to the highest lifting standard in the continuous drought process. The summer and autumn and winter droughts have determined the level algorithm as shown in Table 1.

\section{Results and Analysis}

\subsection{Statistics and Analysis of Drought Process}

According to the daily precipitation data, combined with the classification of the single-station meteorological drought process, the evaluation method of the single 
Table 1. Grade algorithm for continuous drought process over three seasons.

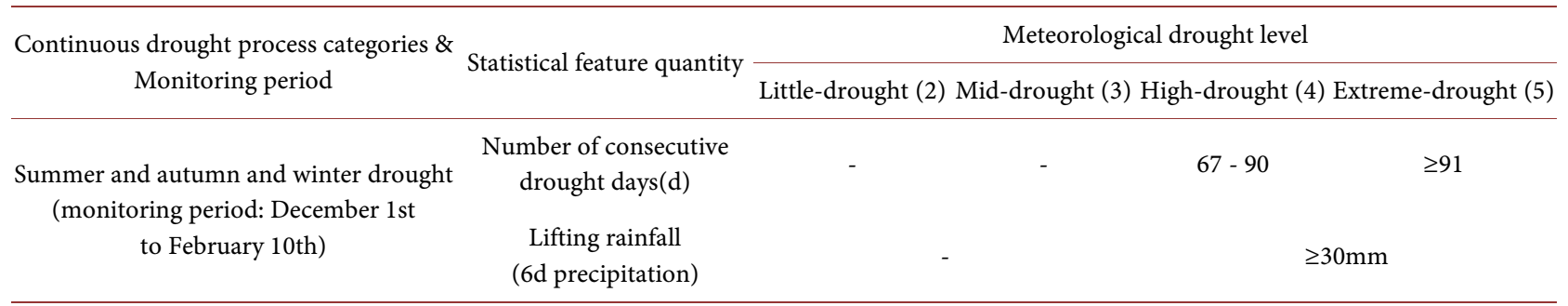

station continuous drought process, the grade of the single station drought process and the grade algorithm of the three-season drought process (Table 1), based on The effective rainfall days and the rainfall release index (DB35/T1283-2012), the meteorological drought process in Pingtan from 1971 to 2016 was calculated. The statistical results show that from 1971 to 2016, the number of meteorological droughts in Pingtan reached 155 (excluding drought), with an average annual drought of 3.3, and there was at least one drought process every year, and the frequency of drought in the second half was much higher than that in the first half.

According to the statistical results (Figure 1), the number of drought days in Pingtan from 1971 to 2016 exceeded 8 days, accounting for 17\%, of which 252 days were the largest in 1976 and 248 days in 2004 and 2005 respectively. 236 days ranked second and third, followed by 246 days in 2003, and the number of drought days in 2004 reached 245 days, and 12 of them contained trans-annual drought. There are 12 times in the process of more than 100 days of drought, and 2 times in more than 200 days.

The longest history is 240 days from August 25, 1999 to April 20, 2000 (Figure 2), 20036 From the 28th of the month to the 2nd of February, the 220th day of the second day, and 9 times is the drought of the New Year. The statistical results show (Figure 2) that there are 45 spring droughts in the statistical period, of which 4 have reached the level of extreme drought; in 64 summer droughts, 11 have reached extreme drought; 21 have autumn and winter drought, no special drought; There were 32 consecutive droughts, including 20 extreme-drought levels. That is to say, in the 35 droughts that reached the extreme drought level, the multi-season continuous drought accounted for 57\%, and the summer special drought 11 times accounted for $31 \%$. The multi-season continuous drought includes $80 \%$ of the summer and autumn seasons, and the first two of the longest duration of the single drought process all appeared in the 00s, including at least the summer, autumn and winter seasons. It can be seen that Pingtan is prone to drought throughout the year, and it is prone to prolonged drought in the second half of the year. Inter-season droughts occur frequently and last long. Autumn and winter droughts generally show longer periods of inter-season or cross-year drought.

Taking the sliding average of 5 years as a cycle, as shown in Figure 1, the number of meteorological drought days in Pingtan is fluctuating: the number of 


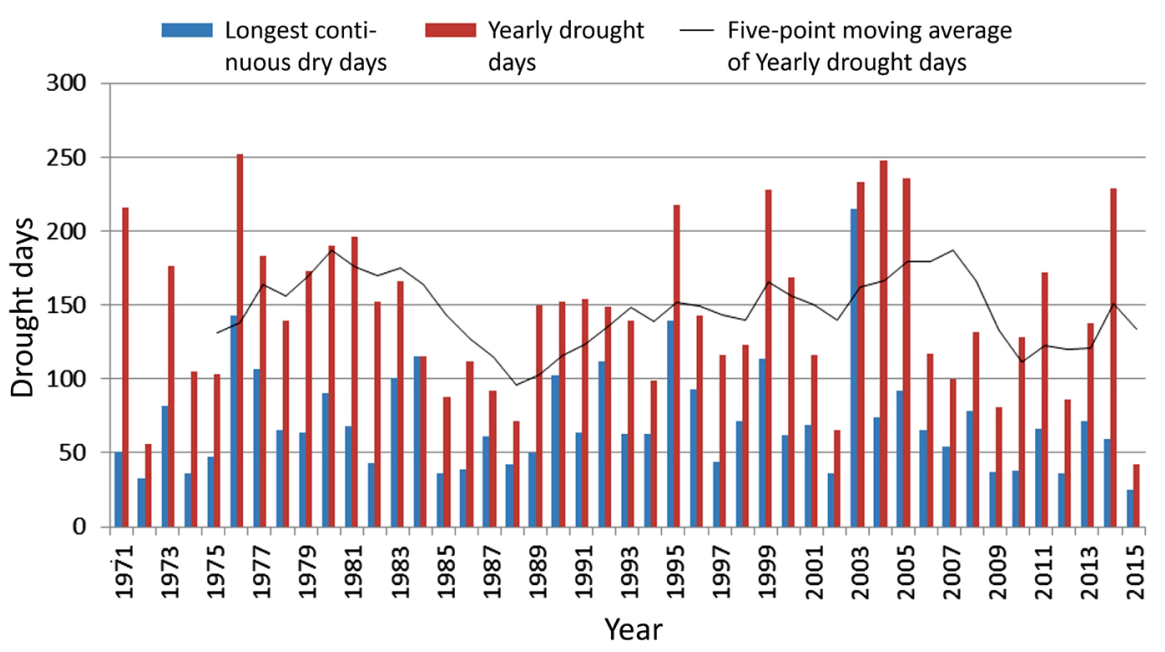

Figure 1. Histogram of meteorological days in Pingtan from 1971 to 2016.

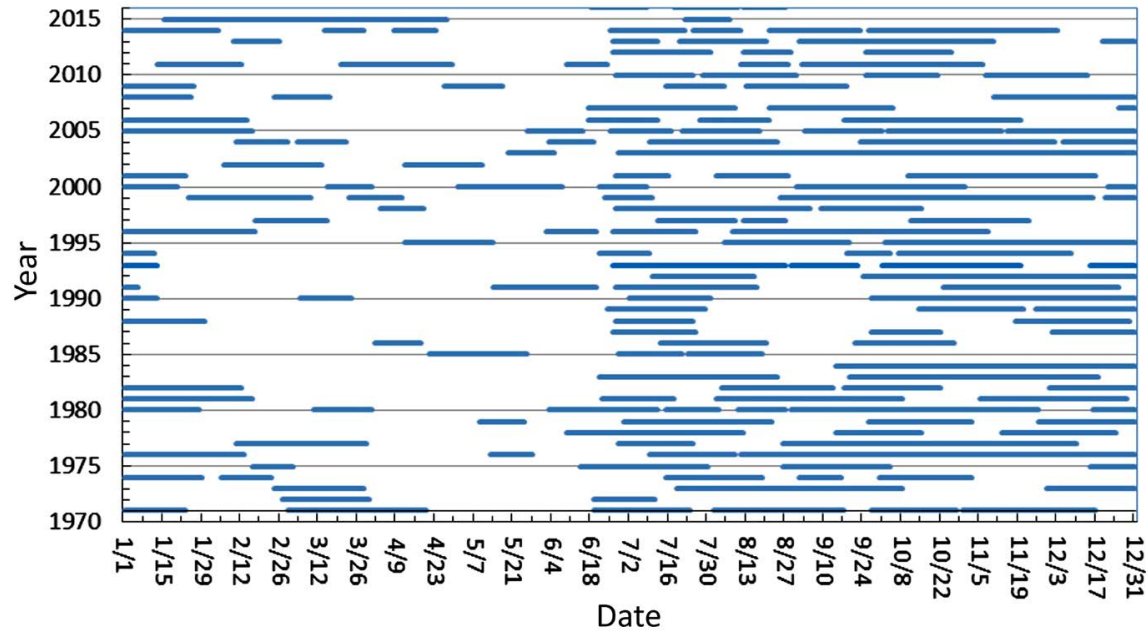

Figure 2. Meteorological drought statistics in Pingtan from 1971 to 2016 (each line length is a drought process duration).

drought days in the 1970s showed a clear upward trend, and in the mid-1980s it decreased significantly. In the late 1980s, it rose sharply. By the end of the 1990s, the number of drought days was maintained at around 150 days. In the middle of the first two years, the number of days was extremely high. In the middle and early 00s (2003-2005), the number of drought days was extremely high and the number of drought days in the next year was the highest. The pattern has decreased and has stabilized for five consecutive years. There has been a slight fluctuation since the 1970s, and the fourth peak appeared in 2014. The chronological distribution of meteorological drought in Pingtan has similar characteristics: the number of special droughts in the 1970s accounted for about $31 \%$, and in the 1980s it dropped sharply to about $14 \%$. In the 1990s, it rose to around $20 \%$. In the 00 s, it is about $23 \%$ of the second peak so far, and it fell back to $12 \%$ in the 10th.

Choose the first two drought years (1999-2000, 2003) and the first-season 
drought days (1973, 2011, 2008-2009) with 6 consecutive drought years. Analysis and classification are carried out, and typical spring and dry circulation types, typical summer and dry circulation types, typical autumn and winter dry circulation types and typical multi-season continuous drought circulation types are obtained.

Comparing the meteorological drought situation in Pingtan with the climate of Fujian, it is found that the drought year in Pingtan is not completely consistent with the overall drought and flood situation in Fujian. The most typical examples are as follows: The year of 1971 is a typical spring drought year in Pingtan, which is consistent with the situation of precipitation and drought in the same period in Fujian. The summer drought in 2003 was the second longest drought days in the statistical period. In the same year, Fujian Province suffered from rare four seasons of drought, and the history of drought loss was rare. In the summer of 1991, the average precipitation in all counties and cities in Fujian Province reached the minimum value from 1961 to 2010, but the drought from Pingtan was only a relatively common year in summer drought. The year of 1973 is a typical summer drought year in Pingtan, while Fujian is a rainy year, and the drought and flood situation is completely opposite, indicating that Pingtan meteorological drought has a strong locality.

\subsection{Localization Correction of Numerical Forecast Products}

Because the precipitation factor is a discontinuous variable, it is proposed to obtain the localized correction product of precipitation through the super-aggregate method of multi-mode integrated forecasting. The rainfall value of each numerical product is used as a factor, and multiple regression is used to predict precipitation. The live value is the 24-hour precipitation (R24) of the day 20-20h. The factor is the 24-hour rainfall forecast for each numerical forecast. The regression coefficient of each factor is first stored in the file using the data of one year. The coefficient file is shown in Figure 3. During the forecast, the amount of precipitation interpolated to each station is multiplied by the coefficient, and the products are added to obtain the predicted value. Figure 4 is the evaluation of the subjective and objective product forecast results from January 24 to 120, 2014. The FJ enQPF indicated by the blue arrow in Figure 4 is the product, the first five items are subjective products, and the last seven items are objective products. The results have a certain reference.

\subsection{Meteorological Drought Warning}

Based on the definition of meteorological drought process by landmarks, based on the above research, a single station meteorological drought warning method is obtained: no effective rainfall days reach critical value + medium precipitation forecast $=$ drought process warning; spring drought, summer drought, autumn and winter drought or continuous drought The number of days reached the critical value + medium-term precipitation forecast (rainfall reached the standard of 


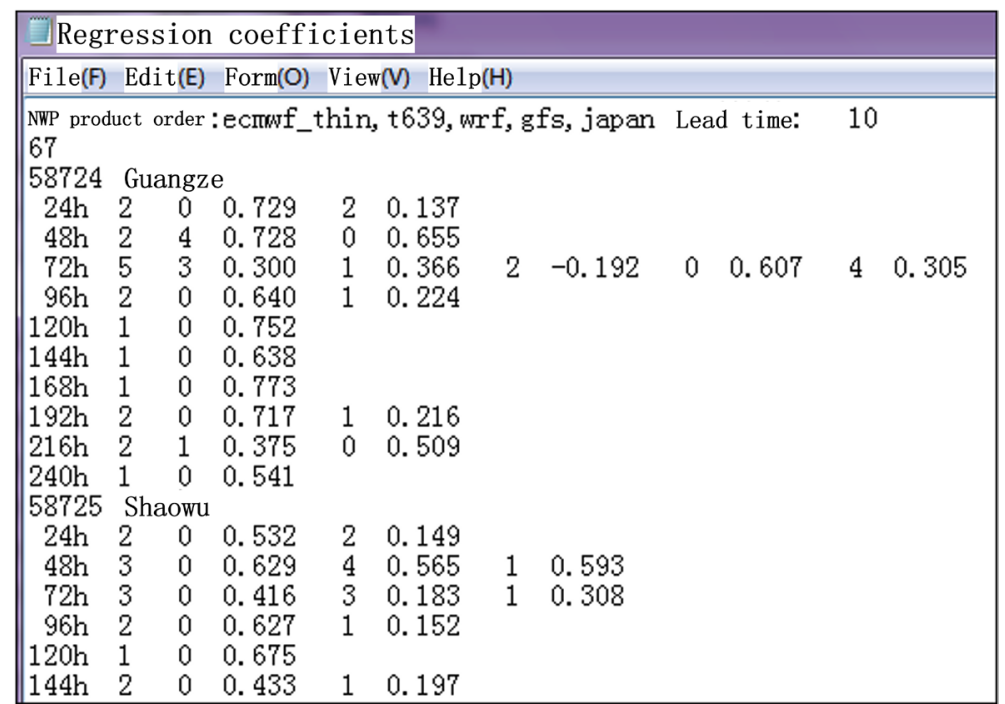

Figure 3. Regression coefficients of multiple regression to prevent and forecast precipitation.

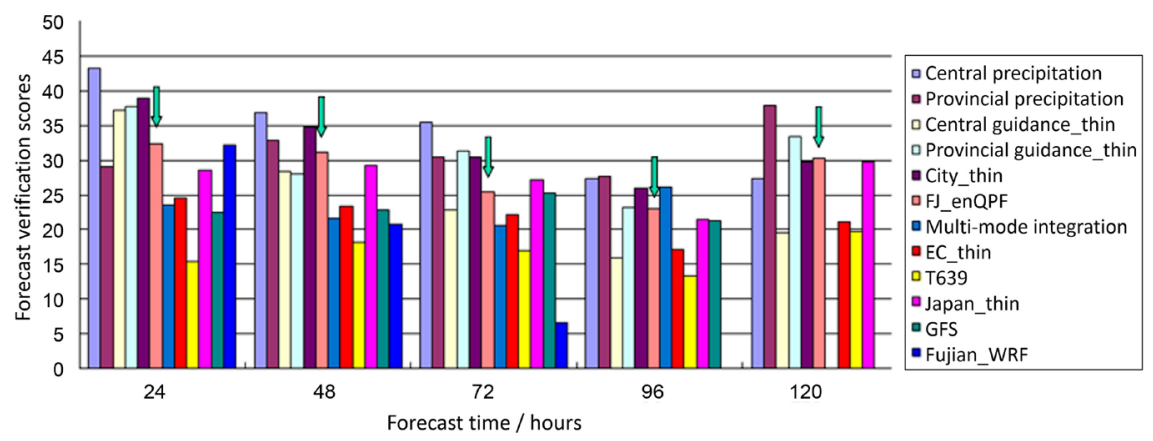

Figure 4. General precipitation verification histogram in January, 2014.

rainfall $)=$ the drought process was lifted. The method is simple in principle, easy to operate or platformize.

The meteorological drought early warning platform must have the function of drought monitoring and forecasting and early warning. At the same time, in order to facilitate future scientific research, the platform should also have the data of the historical data of the drought process.

According to the requirements of the platform, the software function modules of the platform are: Data import function: Import historical precipitation data. The historical data query function of drought: According to the historical precipitation data, the historical drought data is calculated, that is, the drought database is established. Realization: 1) Directly export all historical drought data (Excel format); 2) Query historical drought data and disaster situation according to time, drought type, number of consecutive drought days and circulation type, and then derive specific drought data. The latest drought monitoring and early warning function: judging whether there is drought according to the actual precipitation and short-term and medium-term precipitation forecast; early warning of possible drought in advance; early warning and live monitoring of the 
current drought.

\section{Conclusion and Discussion}

Based on the revised meteorological drought assessment method and single station historical precipitation, this paper statistically analyzed the historical meteorological drought process of single station; through the multi-model integrated forecasting super-aggregation method, studied the precipitation correction method of numerical forecast products; to draw precipitation based on forecasting of the early warning method of single station meteorological drought process, and proposed platformization ideas. The single-station meteorological drought process early warning method is simple in principle, easy to operate after platformization, and can quickly remind decision-making meteorological service personnel to pay attention to the meteorological drought process, and provide platform and data support for decision-making meteorological services.

However, the meteorological drought early warning method studied in this paper is limited to one station and a period of time. This method may have shortcomings and challenges upon the applicability of the model to other geographical regions of similar climatic and water precipitation conditions, can be succinctly given. We also want to make our model more suitable for long-term serial drought statistics, so we will conduct the next step to anticipate specifying the precision, accuracy, consistency and predictability of our model in specific future time of fitting. In future, it is necessary to improve the method of forecasting and warning of a wider range of meteorological drought processes. The methods needed for the large-scale applicability of the model in reality can be used. Many new indices can be used (Wambua et al., 2018), and some tele-connections can also be discovered and utilized (Zeng et al., 2019). From the perspective of large-scale applicability, the government can establish a near-season meteorological drought monitoring and early warning system for cultivated land (Takeuchi et al., 2015).

\section{Acknowledgements}

This work was funded by Fujian Provincial Local Standards Project (DB35/T 1723-2017) of China.

\section{Conflicts of Interest}

The authors declare no conflicts of interest regarding the publication of this paper.

\section{References}

Chen, J. M., \& Zhao, X. C. (2012). Analysis on Drought Characteristics and Indicators in Wuhai City. Journal of Northern Agriculture, 1, 79-81.

Du, L. T., Tian, Q. J., Huang, Y. et al. (2012). Drought Monitoring and Reliability Test in Shandong Province Based on TRMM Data. Journal of Agricultural Engineering, 28, 121-126. 
Duan, H. X., Wang, S. P., \& Feng, J. Y. (2012). The National Drought Situation in 2011 and Its Impact and Causes. Arid Meteorology, 30, 136-147.

Li, X. Y. (2012). Study on the Early Warning Method of Drought in Ningxia. Nanjing: Nanjing University of Information Science and Technology.

Li, Z. G., Zhu, L. L., Cheng, K. K. et al. (2012). Characteristics and Causes of Drought Disasters in Henan Province from 1950 to 2009. Arigricultural of Hubei, 51, 1107-1111.

Liu, Z. X., \& Xiao, Y. (2012). Analysis of Drought and Flood Indexes and Their Change Characteristics in the Upper Reaches. Resources and Environment in the Yangtze River Basin, 21, 210-211.

Takeuchi, W., Darmawan, S., Shofiyati, R., Khiem, M. V., \& Heng, S. (2015). Near-Real Time Meteorological Drought Monitoring and Early Warning System for Croplands in Asia. ACRS 2015: The 36th Asian Conference on Remote Sensing "Fostering Resilient Growth in Asia”.

Wambua, R. M., Mutua, B. M., \& Raude, J. M. (2018). Detection of Spatial, Temporal and Trend of Meteorological Drought Using Standardized Precipitation Index (SPI) and Effective Drought Index (EDI) in the Upper Tana River Basin, Kenya. Open Journal of Modern Hydrology, 8, 83-100. https://doi.org/10.4236/ojmh.2018.83007

Yan, M. C., Zhang, X. C., \& Hu, S. (2012). Drought Monitoring Research in BeijingTianjin-Tangshan Region Based on MODIS Data. Geography and Geographic Information Science, 28, 94-98.

Zeng, L., Schmitt, R. W., Li, L., Wang, Q., \& Wang, D. (2019). Forecast of Summer Precipitation in the Yangtze River Valley Based on South China Sea Springtime Sea Surface Salinity. Climate Dynamics, 15, 1-15. https://doi.org/10.1007/s00382-019-04878-y 